\title{
«IL FAUT QUE J'EXPLIQUE TOUT ÇA». ÉLÉMENTS POUR UNE APPROCHE SOCIOLOGIQUE DE L'EUVRE D'ANNIE ERNAUX
}

\begin{abstract}
Lis Jerzy, "ll faut que j'explique tout ça». Éléments pour une approche sociologique de l'auvre d'Annie Ernaux [,I must explain it". Elements for a sociological approach of Annie Ernaux's works"]. Studia Romanica Posnaniensia, Adam Mickiewicz University Press, Poznań, vol. XXXI: 2004, pp. 59-68. ISBN 83-232-1353-4, ISSN 0137-2475.
\end{abstract}

Annie Ernaux's works, treated by critics as autobiographical, are an original undertaking at writing, which consists in the incessant search of balance between that which in man's life appears as obvious and clcar and that which remains mysterious, disturbing and incomprehensible. The awareness of the dichotomy of the surrounding world and people is a germ of the creative project in the triple perspective - existential, literary and sociological.

Dans la relation bouleversante de l'avortement faite par Annie Emaux dans L'événement, il est question de la mémoire primitive qui «(...) nous fait voir toute la vie passée sous la forme élémentaire de l'ombre et de la lumière, du jour et de la nuit (...)» (p. 74) ${ }^{1}$. L'autobiographe a ainsi rappelé aux lecteurs une vérité on ne peut plus banale: l'existence humaine n'est perçue avec le temps que comme une série d'expériences positives et négatives auxquelles il faut donner un sens. Or, l'acte même d'écrire, du moins dans le cas des autobiographes consiste à rechercher la vérité à travers des événements passés aussi incompréhensibles que contradictoires. La vie humaine, matière élémentaire de tout autobiographe doit donc être considérée non seulement comme une alternance de l'ombre et de la lumière, mais surtout comme une recherche d'un équilibre entre ce qui nous paraît évident et clair et ce qui reste mystérieux, illisible et inquiétant.

Annie Ernaux n'est certainement pas un cas exceptionnel, loin de là. Tout écrivain puise son inspiration dans la nature dichotomique du monde, des hommes et des choses. Autrement dit, la littérature est possible à condition que l'écrivain soit

${ }^{\prime}$ A. Ernaux, L'événement, Gallimard, Paris 2000. La pagination renvoie à la collection Folio, $\mathrm{N}^{\circ} 3556$, édition de 2001. 
capable de saisir la tension entre les contraires et de rendre compte du caractère conflictuel de la relation de l'homme au monde. Dans cette perspective l'écriture d'Annie Enaux pourrait être considérée comme un exemple original de la prise de conscience par des écrivains de la persistance conceptuelle du clair-obscur. En effet, l'auteur de La place a une prédilection marquée pour des confrontations et des comparaisons, et elle fait de la conscience des contraires son credo, réalisé comme un projet artistique à trois dimensions - existentielle, littéraire et sociologique.

Puisque dans le cas d'Annie Ernaux nous avons affaire à une écriture autobiographique, les trois dimensions du projet se réfèrent à une entreprise d'autoanalyse. Du point de vue de l'écriture de soi la question du clair-obscur est d'importance capitale, car elle est à l'origine de tout questionnement à propos de l'évolution de la personnalité. Comme on le sait, le geste autobiographique n'est autre chose qu'un besoin d'expliquer, d'élucider ce qui est sombre. C'est ainsi qu'avant de comprendre l'essentielle dichotomie qui le constitue, l'autobiographe doit tout d'abord effectuer un long trajet qui va de l'ombre à la lumière. Il ne fait pas de doute que l'œuvre littéraire qu'elle soit fictionnelle ou référentielle, consiste à décrire les modes du passage de l'obscurité à la clarté. L'accent, souvent excessif, porté sur le côté sombre ou négatif de cette opposition binaire relève du paradoxe du clair-obscur. La clarté telle quelle ne se décrit pas. Elle ne prend sens que si elle voisine avec le sombre. Il est significatif que dans le corpus de textes autobiographiques on ne trouve presque pas de récits qui relatent le bonheur, la bonté ou la beauté (à une exception près pourtant - Stendhal!). Dans le récit de soi il s'agit de saisir le lien entre le clair et l'obscur ou plus exactement de démontrer dans la perspective temporelle que le sombre est inséparable du clair. L'essence de l'écriture autobiographique réside dans cette espèce d'étonnement qui nous oblige finalement à chercher un équilibre entre ces deux tendances opposées. Tel est le sens du propos d'Annie Ernaux cité ci-dessus.

La dimension existentielle du projet ernalien nous apprend énormément non seulement sur les motivations de la création littéraire, mais aussi sur la nécessité de répondre à des questions concernant la vie humaine. Les trois premiers textes (Les Armoires vides (1974), Ce qu'ils disent ou rien (1977) et La femme gelée (1981)), rédigés par l'écrivain comme des romans traditionnels, correspondent à des moments précis de crises personnelles. Les histoires respectives d'une étudiante, d'une jeune fille de 15 ans et d'une femme mariée sont bien des fictions, mais elles relatent l'existence véridique de la femme qui a réussi à changer de classe sociale: une fille révoltée du milieu ouvrier et paysan est devenue bourgeoise. Racontées au présent, les trois histoires ont pour but de comprendre ce qui s'est passé dans la vie de la femme et de décrire le monde qui n'est plus le sien. Or, l'existence passée est analysée comme une recherche constante de l'équilibre entre la famille et l'école, entre la ville et la campagne, entre la laideur du milieu ouvrier et la beauté des quartiers riches de grandes villes. L'amour des parents est vécu par la fille inséparablement de la haine de leur milieu. Du point de vue existentiel l'équilibre 
des contraires est difficilement subi, car à la fragile clarté correspond la part sombre de ses expériences, celle surtout qui est à l'origine de la honte sociale.

Les trois récits fictifs mettent pourtant l'açcent sur l'instabilité de cet équilibre, évalué avec le temps comme apparent. Revisité bien des années après, le monde d'autrefois reste toujours incompréhensible. Denise Lesur des Armoires vides ne comprend pas la langue de son milieu d'origine, Anne du récit Ce qu'ils disent ou rien se sent étrangère du monde où elle vit, et l'héroïne anonyme du troisième récit (La femme gelée) n'arrive pas à saisir l'existence d'une femme simple. Les trois narratrices procèdent à une nouvelle évaluation des choses, à une autre hiérarchisation, à cette espèce de mise en ordre qui caractérise les récits de vie. C'est d'autant plus intéressant qu'avec la publication en 1983 de La place, l'auteur a renoncé à l'exploitation métaphorique de la déchirure sociale et elle a de nouveau changé de procédures d'élucidation. La fiction a cédé la place à l'écriture plate, laquelle a été mieux adaptée à l'explication de la vie de son père, sujet principal du texte.

La décision prise par Annie Ernaux de parler ouvertement de sa biographie a été la conséquence naturelle d'une révélation qu'il n'est plus possible d'occulter les ambiguiités du clair-obscur existentiel. L'écrivain mettra désormais en relief ce qu'elle nomme l'effroi de la réalité vécue. Des choses indicibles et honteuses seront appelées par leur nom, et la partie sombre et cachée de l'existence aura droit de cité dans les textes ${ }^{2}$. Du point de vue du projet littéraire Emaux a bouleversé l'ordre des choses. Si dans les œuvres de fiction elle explorait le drame de la déchirure sociale (la part sombre et négative de son existence), dans les œuvres dites autobiographiques, l'écrivain amorce un projet très réfléchi de renouer avec sa famille et son milieu d'origine. La mise au jour des expériences vécues par elle devient un devoir et un manifeste à caractère social ${ }^{3}$. Mais il y a encore une autre chose importante qui explique pourquoi le mode d'écriture a changé avec le quatrième livre d'Ernaux. Dans les trois premiers romans, les questions ont été posées, mais les textes manquaient d'authenticité, comme si les références explicites à la réalité exigeaient d'autres supports et d'autres méthodes. Ernaux revient à ce sujet dans une interview où elle souligne le rapport étroit de l'écriture avec l'évocation de la réalité quasi-indicible ${ }^{4}$. Être authentique ne veut pas dire se mettre à nu. D'ailleurs, l'écrivain ne fera pas de cette authenticité le marché de l'intimité, comme elle sera également loin du narcissisme typique des écritures de soi ${ }^{5}$.

${ }^{2}$ Ph. Vilain, Interview avec Annie Ernaux, Annie Ernaux ou l'autobiographie en question, «Roman 50/90», $\mathrm{N}^{\circ} 24,1997$, pp.141-147.

${ }^{3} \mathrm{M}$. Boehringer, Tombeau d'une mère: «elle» e(s)t «je»: Une femme et «Je ne suis pas sortie de ma nuit» d'Annie Ernaux, dans: M. Boehringer (dir.), Écriture de soi au féminin, «Dalhousie French Studies» (Spécial Issue), N $^{\circ} 47,1999$, pp. 155-163. Cf. aussi M. Boehringer, Écrire le dedans et le dehors: dialogue transatlantique avec Annie Ernaux, dans le même numéro, pp.165-170.

4 S. Laacher, Annie Ernaux ou l'inaccessible quiétude, suivi d'un entretien avec l'écrivain, «Politix. Travaux de science politique», $\mathrm{N}^{\circ} 14,1991$, pp. 73-78.

${ }^{5} \mathrm{Cf}$. à ce propos $\mathrm{G}$. Lipovetsky, L'ère du vide. L'essai sur l'individualisme contemporain, éd. Gallimard, Paris 1983. Voir surtout le chapitre III: «Narcisse ou la stratégie du vide». 
La revendication de sa propre appartenance sociale aura été donc un moyen de chercher une autre forme d'équilibre entre l'obscur et le clair. Appelée par l'écrivain «certitude» ou «illumination», cette conscience désigne à la fois partage du paradigme de quotidienneté de ses parents et considération de l'existence en termes de pratique béhavioriste. Ne sont relatés que les faits, les événements et non pas les pensées des parents, comme ne sont citées que les expressions de tous les jours et non pas les réflexions abstraites. Ce principe adopté par Annie Ernaux dans $L a$ place l'obligera à penser l'existence passée comme une série de preuves à donner. Ainsi pour décrire son père, elle ne peut pas se servir de récit, mais: «(...) Je rassemblerai les paroles, les gestes, les goûts de mon père, les faits marquants de sa vie, tous les signes objectifs d'une existence que j'ai aussi partagée» (p. 24) ${ }^{6}$. Elle réunira aussi des souvenirs tels qu'ils se sont gravés dans sa mémoire, des arrêts sur image évocateurs de l'infériorité d'autrefois, et aujourd'hui - objets de revalorisation. Dans Une femme ${ }^{7}$ elle va encore plus loin en citant plusieurs séries d'images de sa mère, saisie dans différentes circonstances, souvent obscures et honteuses.

Dans les œuvres postérieures, l'exploitation des faits biographiques prendra un caractère minimaliste, car à la description Ernaux préférera, les registres, les

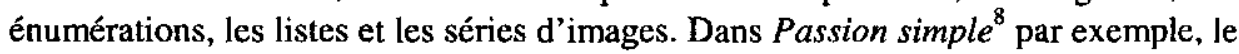
côté sombre de la relation amoureuse est représenté par des citations explicites des preuves, c'est-à-dire des occupations pendant l'attente de son amant. L'autobiographe expose les choses sans les expliquer comme si elle voulait que l'inventaire des travaux quotidiens remplace le récit systématique et se substitue au registre romanesque. En même temps la narratrice affirme son identité en accumulant les signes d'une passion qui sont de nature différente: journaux feuilletés, horoscopes étudiés, film regardés, etc. Or, l'élucidation du caractère contradictoire des expériences vécues se fait par l'intermédiaire du présent qui sert de filtre lors de l'évaluation de la vie passée. Il s'agit au fond de «(...) forcer le présent à redevenir du passé ouvert sur le bonheur (...)" (Passion simple, p. 58).

En évoquant la dimension existentielle de l'écriture, nous sommes parfaitement conscient que quelle que soit la forme d'expression, il s'agit toujours de l'élucidation de ce qui est ou était inquiétant et mystérieux. C'est en cela d'ailleurs que consiste l'un des paradoxes de l'écriture autobiographique - l'auteur cherche un langage capable d'exprimer les dilemmes du clair-obscur existentiel. Le mode de dévoilement dépend bien entendu du projet et du caractère des expériences vécues. L'œuvre d'Annie Ernaux est un bon exemple de ce type d'écriture où il s'agit d'approcher de diverses manières les choses obscures. Puisque dans les trois romans l'expression fictive de la biographie d'une femme n'a pas permis de

${ }^{6}$ A. Ernaux, La place, Gallimard, Paris 1983. La pagination renvoie à la collection Folio, $N^{*}$ 1722 (éd. 1986).

'Eadem, Une femme, Gallimard, Paris 1987. Pour les citations nous utilisons l'édition de poche, collection Folio, $\mathrm{N}^{\circ} 2121$ (1992).

${ }^{8}$ Eadem, Passion simple, Gallimard 1991 (les citations sont tirêes de l'édition originale). 
résoudre les problèmes à caractère social, l'écrivain abandonne la forme romanesque pour parler d'une autre façon de la femme (c'est toujours la même femme) issue du milieu pauvre des petits commerçants. Le rejet ostentatoire de la fiction à partir de La place symbolise la mort définitive du personnage fictif. Désormais l'écriture romanesque et la fiction en particulier, sera considérée comme synonyme de mensonge et elle ne pourra plus servir le projet qu'elle met en ouvre ${ }^{9}$. On voit dans cette attitude le désir explicite de transgresser les valeurs symboliques de la littérature. Les noms fictifs disparaîtront des œuvres comme seront accentuées toutes les références à la réalité historique ou quotidienne. Le récit cèdera la place à une description ou bien à une série de réflexions sans parler d'archivisation des faits, de cette archivisation mnémotechnique qui est devenue l'un des traits distinctifs de l'écriture ernalienne ${ }^{10}$.

En adoptant l'écriture plate, Ernaux crée les cadres d'une relation quasi scientifique à la limite du sociologique et de l'ethnologique où la clarté et la transparence deviennent un objectif élémentaire. En même temps la démarche de ce type impose à l'écrivain une rigueur d'ordre factuel grâce à quoi les événements racontés sont situés précisément dans le contexte spatio-temporel. Le langage simplifié à l'extrême est selon Ernaux non seulement mieux adapté à l'expression des vérités essentielles, mais il est un moyen efficace de maîtriser les obscurités d'autrefois. De manière générale et sans que cela soit une qualité exceptionnelle de l'écrivain, le besoin de la précision, de la simplicité et de la brièveté équivaut à la recherche de la clarté et de la lumière. Avec la pratique de l'écriture plate Annie Ernaux est arrivée aussi à concevoir le monde selon les règles précises et claires. Ainsi par exemple dans Passion simple au récit chronologique de sa liaison avec un diplomate russe l'écrivain préfère un compte-rendu d'absences et de présences de l'amant, censé exprimer le mieux la vérité de cette relation particulière (cf. Passion simple, pp. 31-34).

Les réflexions autothématiques qui abondent dans les textes d'Ernaux, permettent de mesurer l'importance de la littérature dans l'éclaircissement de cette obscurité qu'est le passé d'une famille de province. Renouer avec le milieu d'origine et surtout avec ses propres parents, c'est en finir avec la trahison et la honte sociale à l'aide d'une écriture: une phrase Il faut que j'explique tout ça ( $L a$ place, p. 23) résonne dans les textes qui illustrent la recherche d'un ordre idéal dans l'existence humaine. Avec sa manie d'archiviste, Emaux accumule et regroupe, souvent par association, les images, les gestes, les expressions utilisées par ses parents et les gens croisés dans les années cinquante et soixante. C'est par ce travail minutieux qu'elle peut espérer un effet de dévoilement, mais pour obtenir un résultat satisfaisant, outre l'exploration de sa propre mémoire, il lui faut également s'appuyer sur l'observation minutieuse de la réalité présente. C'est là que l'écrivain retrouve le filtre indispensable pour une interprétation des scènes et des faits de son

${ }^{9}$ M.-F. Savéan (commente), «La place» et «Une femme» d'Annie Ernaux, Gallimard, coll. Foliothèque, $\mathrm{N}^{\circ} 36$, Paris 1994, pp. 182 et suivantes.

${ }^{10}$ Cf. B. Owczarek, Poetyka powieści niefabularnej, PWN, Warszawa 1999, p. 12. 
propre passé. Il s'agit toujours des images que la mémoire a retenues. Parfois ce sont des scènes sans grande valeur factuelle, mais qui du point de vue des émotions sont devenues indélébiles. Pour comprendre leur sens, Ernaux cherche dans la vie présente des équivalences ou des signes de ressemblance entre différents phénomènes et images: «(...) C'est dans la manière dont les gens s'assoient et s'ennuient dans les salles d'attente, interpellent leurs enfants, font au revoir sur les quais de gare que j'ai cherché la figure de mon père. J'ai retrouvé dans des êtres anonymes rencontrés n'importe où, porteurs à leur insu des signes de force ou d'humiliation, la réalité oubliée de sa condition» (La place, pp. 100-101).

Dans les textes postérieurs à La place les observations de la vie présente seront évaluées dans la perspective des traces émotionnelles que laissent les expériences passées. On le voit déjà très bien dans le texte suivant Une femme où Annie Ernaux s'impose une tâche difficile de relier deux images opposées de sa mère: «(...) Je sais que je ne peux pas vivre sans unir par l'écriture la femme démente qu'elle est devenue, à celle forte et lumineuse qu'elle avait été» (p. 89). L'écriture en tant que recherche de la clarté consiste selon l'écrivain à comprendre le sens des choses en juxtaposant ce qui est à priori incomparable ou incompatible ${ }^{11}$. Ce type d'association est fréquent dans la plupart des textes ernaliens: des objets énumérés s'associent aux gestes ou attitudes dans la même mesure que des sculptures ou des tableaux de peinture peuvent servir d'illustration à tels ou autres moments de notre existence (cf. Passion simple, pp. 20 et 40). Le rapprochement des contraires ou encore l'explication par juxtaposition des situations incomparables donnent souvent des résultats surprenants. La narratrice de Passion simple réfléchit sur une liaison implicite entre la puissance du sentiment d'amour et le conflit en Iraq (sic!). L'avortement raconté dans L'événement est rapproché de l'assassinat du président Kennedy. La honte personnelle relatée dans le texte qui a le même titre est reliée à la honte médiatique à propos de Saraïevo. Des exemples sont multiples: le dépistage du sida rappelle le drame de l'avortement, le meurtre manqué de sa mère par le père fait penser au bruit de la course automobile du Mans. Il arrive souvent que l'association des faits différents n'est qu'apparence et illusion comme c'est le cas de L'occupation ${ }^{12}$ où le sentiment de la jalousie (l'occupation de l'esprit par la jalousie) est vécu parallèlement au crash du Concorde près de l'aéroport de Roissy.

Les associations forgées par Emaux ne se limitent pas seulement à l'événementiel. Dans La Honte ${ }^{13}$ la narratrice juxtapose des photographies censées visualiser deux étapes opposées de son existence: la fin de l'enfance et le début de la période de la honte sociale. Il est à remarquer que tous les textes avancent grâce au travail de juxtaposition non seulement des événements, mais aussi des paroles et des

"S. Marson, Women on Women and the Middle Man: Narrative Structures in Duras and Emaux, "French Forum", the University of Nebraska Press, vol. 26, $\mathrm{N}^{\mathrm{D}}$ 1, Winter 2001, pp. 67-82.

${ }^{12}$ A. Ernaux, L'occupation, Gallimard , Paris 2002.

${ }^{13}$ Eadem, La honte, Gallimard, Paris 1997. L'édition citée est celle de la collection Folio, N³154 (éd. 2001). 
gestes qui appartiennent aux époques différentes. On peut distinguer deux stratégies qu'Annie Enaux met en pratique dans l'ensemble de ses textes: soit l'existence passée est décrite à l'aide du langage d'autrefois, soit, faute de mémoire, c'est le langage courant qui sert de moyen pour une interprétation des choses révolues. $\mathrm{La}$ première stratégie pourrait être résumée par une citation tirée de La Honte: «(...) Ce qui m'importe, c'est de retrouver les mots avec lesquels je me pensais et pensais le monde autour. Dire ce qu'étaient pour moi le normal et l'inadmissible, l'impensable même. (...) Il n'y a pas de vraie mémoire de soi. Pour atteindre ma réalité d'alors, je n'ai pas d'autre moyen sûr que de rechercher les lois et les rites, les croyances et les valeurs qui définissaient les milieux, l'école, la famille, la province, où j'étais prise et qui dirigeaient, sans que j'en perçoive les contradictions, ma vie. (...)» (p. 39-40) Les listes des interdictions et des rites d'autrefois, les inventaires des codes sociaux et moraux de l'époque ne font que ranimer le langage des Français de province des années cinquante. Lorsque la mémoire et la documentation font défaut, Annie Ernaux recourt à une autre stratégie qui consiste à expliquer les pages sombres de sa vie à l'aide du langage des passagers du métro ou des passants croisés dans la rue: «(..) noter les gestes, les attitudes, les paroles de gens que je rencontre me donne l'illusion d'être proche d'eux. Je ne leur parle pas, je les regarde et les écoute seulement. (...) Peut-être que je cherche quelque chose sur moi à travers eux, leurs façons de se tenir, leurs conversations (...)» (Journal du dehors, p. 36-37). Force est de convenir que le langage manié par l'écrivain, le français standard notamment, reflète la réalité quotidienne et il est en même temps une source des sensations physiques. En renonçant au récit traditionnel, Ernaux enregistre des rencontres et des instants vécus dans la rue ou dans le métro en suivant les techniques de l'observation participante propre aux sciences sociales. Faire de l'ethnotexte, c'est mettre en ordre des images et des gestes à l'aide des mots qui seuls sont en mesure de ressusciter l'événement. Rien ne caractérise mieux le projet ernalien que cet aveu de Michel Leiris placé en exergue de L'événement: Mon double vœu: que l'événement devienne écrit. Et que l'écrit soit événement.

Du point de vue de la production littéraire il importe de signaler encore une autre manière de considérer l'écriture comme une élucidation. Pour en comprendre l'enjeu et surtout la logique, il faut revoir la thématique de son œuvre. Dans les trois premiers romans Annie Ernaux a présenté sous une forme fictive sa propre vie ainsi que tout le contexte familial et social. Les principaux thèmes de son auvre ont été donc signalés. Le lecteur sait à qui il a affaire, quelles sont les aspirations de la jeune fille. Il dispose également d'informations bien précises sur le milieu social de ses parents. Les textes suivants, lesquels, comme nous le savons, ne sont plus des romans, reprennent d'une autre manière certains problèmes dévoilés entre 1974 et 1981, mais Ernaux ne cesse de publier des récits qui se font l'écho de la réalité présente (dont Passion simple (1991), Journal du dehors (1993), La vie extérieure (2000) et L'occupation (2002). Ces livres ont tous un dénominateur commun, formulé par l'écrivain dans Je ne suis pas sortie de ma nuit (1997): un désir de 
sauver et de comprendre (p.108). Un désir double comme double est aussi la tentative d'attaquer le même thème. Trois textes auront désormais leur version brute, appelée soit substrat, soit matériau brut, soit résidus ou encore trace. Aux Armoires vides correspond L'événement publié 26 ans plus tard. L'histoire fictionnalisée aura trouvé un complément sous forme de témoignage où Ernaux raconte les circonstances d'un avortement qu'elle a subi il y a un quart de siècle et dont le dévoilement n'était possible qu'après la mort de sa mère. Le texte Une femme de 1987 sera completé par le journal tenu par Ernaux lors de la maladie de sa mère (maladie d'Alzheimer) publié sous le titre de Je né suis pas sortie de ma nuit (1997). Un autre journal Se perdre paraîtra en 2001 comme un palimpseste de Passion simple ${ }^{14}$. La reprise de la thématique de ses livres témoigne du besoin d'aller au plus profond de l'émotion et d'élucider à fond son existence. C'est la différence entre le «je» romanesque et le «je» autobiographique, la présence de ce «je» ou son absence qui détermine, selon l'écrivain, le degré de vérité et de réalité. La publication de la relation brute de l'avortement et de ces deux journaux permet à Ernaux de donner un autre éclairage des textes rédigés auparavant. Ils peuvent être considérés comme des supports crus, directs et beaucoup plus violents des autres textes $^{15}$. A la relation construite au passé se superpose donc une autre version aussi immédiate que provisoire, et c'est dans ce rapprochement de deux modes d'écriture relatifs soit au passé révolu, soit à la réalité présente que l'écrivain vit un moment privilégié de l'illumination et de la certitude à propos des changements qui ont eu lieu dans sa vie.

L'habitude de «foumir toutes les pièces» (expression de Rousseau), laquelle serait une manière particulière de passer de l'ombre à la lumière, permet d'adoucir les effets indésirables de l'écriture de soi et de se concevoir dans la perspective plus large, celle de l'autre, indispensable à un équilibre psychique et social de l'individu. Il s'agit d'une attitude d'identification fréquente qui détermine les relations de l'écrivain avec les autres. En même temps, elle caractérise sa démarche scripturale située au croisement de la littérature et des sciences sociales. La dimension sociologique de son projet a été exprimée de manière explicite dans La place et dans Une femme où nous lisons: «(...) ce que j'espère écrire de plus juste se situe sans doute à la jointure du familial et du social, du mythe et de l'histoire. Mon projet est de nature littéraire (...) Mais je souhaite rester, d'une certaine façon, audessous de la littérature» (Une femme, p. 23). Or, l'utilisation des méthodes propres aux sciences sociales lui permet non seulement d'envisager l'explication de la situation économique et sociale de ses parents, mais aussi de se servir des cas particuliers pour généraliser les questions qui l'intéressent. Pour Annie Ernaux,

${ }^{14} \mathrm{Cf}$.Mon écriture cherche à transcrire la violence de la réalité», entretien de Jean-Louis Tallon avec Annie Ernaux, «HorsPresse. Webzine culturel», mars 2001, http://perso.wanadoo.fr/erato/horspress/ernaux.htm; article consulté le 21 octobre 2002.

${ }^{15} \mathrm{Cf}$. A. Ernaux, L'écriture comme un couteau. Entretien avec Frédéric-Yves Jeannet, éd. Stock, Paris 2003, pp. 38-39. 
s'arracher du piège de l'individuel (La place, p. 45) c'est saisir les mécanismes sociaux, c'est-à-dire devenir consciente des différences entre les classes sociales par la description de son existence ou de celle de ses parents dans la perspective sociologique et ethnologique. Pour tout récit ernalien le point de départ ce serait la prise de conscience de deux déterminations: l'injustice sociale (différences des classes) et l'injustice entre les hommes et les femmes. Aussi n'est-il pas étonnant que la littérature prise ici à la lettre comme une recherche de type sociologique soit une entreprise à visée suivante: replonger dans le temps révolu et revivre les événements marquants, les sauver, essayer de les comprendre et finalement agir sur le monde ${ }^{16}$.

Annie Ernaux cherche le matériau pour ses livres au croisement de l'expérience historique et de l'expérience individuelle. Elle instaure le véritable dialogue avec les autres pour saisir les différences sociales qui, comme elle le dit elle-même, structurent une personne ${ }^{17}$. En tant que sociologue et par l'intermédiaire du dialogue avec l'autre, Ernaux déchiffre toutes les expériences qu'elle a pu emmagasiner tout au long de sa vie. Elle le fait en suivant la méthode de Bourdieu même si la spontanéité de son geste ne correspond pas tout à fait à la rigueur scientifique du célèbre sociologue. En même temps, l'autosocioanalyse ernalienne recourt aux méthodes positivistes traditionnelles de la connaissance de l'autre: Annie Ernaux commence par l'examen de l'autre, procède ensuite à sa description, son interprétation et la construction du système ${ }^{18}$.

Il est évident que l'utilisation des méthodes ethnosociologiques n'a été possible qu'après la prise de conscience du changement de classe. Le statut de transfuge lui a facilité l'analyse ethnographique centrée sur l'observation de l'autre et des circonstances de son existence (habitat, déplacements, rythme de vie, lieu de travail, etc.). Cependant pour Annie Ernaux l'enquête socio-ethnologique est avant tout un moyen d'objectivation de son propre passé. Comprendre ce qui s'est passé, ce n'est pas seulement réhabiliter une vie inférieure, mais c'est surtout dénoncer l'aliénation de l'autre et partager sa déréliction. Le sens de l'existence est perceptible à condition que l'identification avec l'autre soit totale. Les exemples ne manquent pas, mais l'histoire anecdotique d'une célèbre religieuse Sœur Dominique dite Sœur Soleil est certainement la plus symptomatique de ce type de relation à l'autre: «(...) C'est donc au-dehors, dans les passagers du métro ou du R.E.R., les gens qui empruntent l'escalator des Galeries Lafayette et d'Auchan, qu'est déposée mon

${ }^{16}$ Cf. C. Arand, Entretien avec Annie Ernaux, «Lire», avril 2000.

${ }^{17} \mathrm{Cf}$. J. Pécheur, Une place à part. Entretien avec Annie Ernaux. Version électronique de l'entretien disponible sur le site internet http://www.fdlm.org/article/3 10/ernaux.htm (article consulté le 21 octobre 2002).

${ }^{18}$ Annie Ernaux n'est pas loin non plus des méthodes propres aux recherches anthropologiques. Au fond, le discours anthropologique n'est pas possible sans l'expérience de l'altérité. Cf. à ce propos Mondher Kilani, L'invention de l'autre. Essais sur le discours anthropologique, éd. Payot, Lausanne 1994. 
existence passée. Dans des individus anonymes qui ne soupçonnent pas qu'ils détiennent une part de mon histoire, dans des visages, des corps, que je ne revois jamais. Sans doute suis-je moi-même, dans la foule des rues et des magasins, porteuse de la vie des autres» (Journal du dehors, pp. 106-107).

La mise en mots de l'expérience humaine totale apparait donc chez Annie Ernaux comme une entreprise d'élucidation de l'obscurité dans le but de «produire» la réalité, cette réalité que composent la mémoire et la conscience sociale. $\mathrm{Si}$ l'écriture (et pas seulement l'écriture autobiographique) consiste à donner une forme à la vérité, les textes de cet écrivain pourraient témoigner en effet des tentatives de saisir ce qu'on cherche comme vérité et ce qui, en même temps, se dérobe sans cesse. Pour Annie Ernaux - qui ne fait que chercher sa propre vérité, il ne saurait y avoir de meilleure solution que l'élucidation par le biais d'un jeu avec les énigmes du clair-obscur. 